\title{
Prevalence of subretinal drusenoid deposits in older persons with and without age-related macular degeneration, by multimodal imaging
}

\author{
Anna V. Zarubina, MD ${ }^{1}$, David C. Neely, MD ${ }^{1}$, Mark E. Clark, BS ${ }^{1}$, Carrie E. Huisingh, \\ MPH $^{1,2}$, Brian C. Samuels, MD, PhD1, Yuhua Zhang, PhD ${ }^{1}$, Gerald McGwin Jr., MS, PhD ${ }^{1,2}$, \\ Cynthia Owsley, PhD, MSPH ${ }^{1}$, and Christine A. Curcio, PhD $^{1}$ \\ ${ }^{1}$ Department of Ophthalmology, School of Medicine, University of Alabama at Birmingham; \\ Birmingham, Alabama \\ 2Department of Epidemiology, School of Public Health, University of Alabama at Birmingham; \\ Birmingham, Alabama
}

\begin{abstract}
Purpose-To assess the prevalence of subretinal drusenoid deposits (SDD) in older adults with healthy maculas and early and intermediate age-related macular degeneration (AMD) using multimodal imaging.

Design-Cross-sectional study.

Participants-A total of 651 subjects aged $\geq 60$ years enrolled in the Alabama Study of Early Age-Related Macular Degeneration from primary care ophthalmology clinics.

Methods-Subjects were imaged using spectral domain optical coherence tomography (SDOCT) of the macula and optic nerve head (ONH), infrared reflectance, fundus autofluorescence, and color fundus photographs (CFP). Eyes were assessed for AMD presence and severity using the AREDS 9-step scale. Criteria for SDD presence were identification on $\geq 1$ en-face modality plus SD-OCT or on $\geq 2$ en-face modalities if absent on SD-OCT. SDD were considered present at the person-level if present in 1 or both eyes.
\end{abstract}

Main outcomes measures-Prevalence of SDD in participants with and without AMD.

Results-Overall prevalence of SDD was 32\% (197/611), with 62\% (122/197) affected in both eyes. Persons with SDD were older than those without SDD (70.6 vs. 68.7 years, $\mathrm{p}=0.0002$ ).

\footnotetext{
Correspondence to: Christine A. Curcio, PhD; University of Alabama at Birmingham, Department of Ophthalmology, 1670 University Boulevard, VH 360, Birmingham, AL, 35294, USA; curcio@uab.edu; Tel: +1 (205) 996 8682; Fax: +1 (205) 9343425.

The abstract was presented at the ARVO Annual Meeting, May 6, 2015, Denver CO.

Conflict of Interest: no conflicting relationship exists for any author.

This article contains additional online-only material. The following should appear online-only: Supplementary tables 1, 2, 5 and Supplementary Figure 4. Supplemental material is available at http://www.aaojournal.org

Publisher's Disclaimer: This is a PDF file of an unedited manuscript that has been accepted for publication. As a service to our customers we are providing this early version of the manuscript. The manuscript will undergo copyediting, typesetting, and review of the resulting proof before it is published in its final citable form. Please note that during the production process errors may be discovered which could affect the content, and all legal disclaimers that apply to the journal pertain.
} 
Prevalence of SDD was 23\% in subjects without AMD and 52\% in subjects with AMD $(\mathrm{p}<0.0001)$. Among those with early and intermediate AMD, SDD prevalence was $49 \%$ and $79 \%$, respectively. After age adjustment, those with SDD were 3.4x more likely to have AMD than those without SDD (95\% CI 2.3-4.9). By using CFP only for SDD detection per the AREDS protocol, prevalence of SDD was 2\% (12/610). Of persons with SDD detected by SD-OCT and confirmed by at least one en-face modality $47 \%$ (89/190) were detected exclusively on the ONH SD-OCT volume.

Conclusion-SDD are present in approximately one quarter of older adults with healthy maculae and in more than half of persons with early to intermediate AMD, even by stringent criteria. The prevalence of SDD is strongly associated with AMD presence and severity and increases with age, and its retinal topography including peripapillary involvement resembles that of rod photoreceptors. Consensus on SDD detection methods is recommended to advance our knowledge of this lesion and its clinical and biologic significance.

\section{Introduction}

Subretinal drusenoid deposits (SDD) are extracellular retinal lesions found between retinal pigment epithelium (RPE) and photoreceptors and localized prominently in the superiortemporal perifovea. ${ }^{1,2}$ Subretinal drusenoid deposits appear to share some proteins (apolipoprotein E, complement factor $\mathrm{H}$ and vitronectin) with sub-RPE drusen and differ markedly from sub-RPE drusen in lipidic composition. ${ }^{3-5}$ Despite their localization to the subretinal space, SDD lack markers for photoreceptors, Müller cells, and RPE, 1,3 and do not contain recognizable outer segment disks. ${ }^{1,6}$ Yet adaptive optics-assisted imaging shows that photoreceptors are perturbed in a stage-specific manner around these deposits, ${ }^{7}$ which can affect visual function. ${ }^{8,9}$ (Neely, D., et al. Association between subretinal drusenoid deposits seen by multimodal imaging and dark adaptation in normal, early, and intermediate age-related macular degeneration eyes. Invest Ophthalmol Vis Sci 2015; 56(7): 2777. ${ }^{10}$ ).

Subretinal drusenooid deposits were originally described as pseudodrusen "visible en lumiere bleue" by blue reflectance photography in 1990. ${ }^{11}$ They were later called "reticular pseudodrusen" because of their appearance in scanning laser ophthalmoscopy as a reticular pattern forming "branches and ill-defined interlacing network". ${ }^{12}$ The name "subretinal drusenoid deposits" was offered because of the location and composition of lesions seen in histopathology. ${ }^{3,13}$ Independently, spectral domain optical coherence tomography (SDOCT) demonstrated reflective features present between the RPE-Bruch's band and the inner segment ellipsoid band, ${ }^{14}$ and the SDD name was adopted from histology. ${ }^{15}$ Thus, terminology evolved in relation to the imaging modality, and prevalence estimates reported for SDD varied both with technology and patient population (Supplementary Table 1, available at http://www.aaojournal.org). ${ }^{2}, 12,14,16-29$ Further the morphological heterogeneity of SDD subtypes is now recognized. ${ }^{30,31}$ There is currently no consensus on the best imaging approach to SDD diagnosis. Many authors recommend using SD-OCT because it allows visualization of hyperreflective material in the subretinal space and infrared reflectance (IR) for screening over a wide area of macula. ${ }^{32}$ Others also used 
fundus autofluorescence (FAF), red-free light, channel-separated color fundus photographs (CFP), and indocyanine green angiography. ${ }^{26,33}$

The clinical significance of SDD has been established most strongly in relation to the incidence and progression of late age-related macular degeneration (AMD), 27, 28, 34, 35 especially type 3 (intraretinal) neovascularization. 35,36 However, SDD also accompany a variety of inherited ${ }^{37-40}$ and acquired disorders. ${ }^{22,41-43}$ Our understanding of SDD significance would be furthered by new epidemiologic data on SDD prevalence in a large number of older adults with healthy maculas. To our knowledge, only two prospective population-based studies evaluated reticular drusen prevalence, using stereoscopic CFP, ${ }^{21,44}$ which has limitations as an SDD detection technology, ${ }^{2}$ and no study has established SDD prevalence in a population with many normal maculas using multimodal retinal imaging. To address this question, we took advantage of the Alabama Study of Early Age-Related Macular Degeneration (ALSTAR), a prospective clinic-based study of older adults with normal macular health and those with early and intermediate AMD. The primary aim of ALSTAR is to examine the association between delayed rod-mediated dark adaptation and incidence or progression of AMD 3 years later. ${ }^{45,46}$ Among ALSTAR study advantages is the availability of SD-OCT scans through the peripapillary area, ${ }^{2,47,48}$ in addition to macula, where SDD were detected previously.

\section{Materials and Methods}

This study was approved by the Institutional Review Board of the University of Alabama at Birmingham and followed the tenets of the Declaration of Helsinki. Written informed consent was obtained from each participant after the nature and purpose of the study were explained.

\section{Study Sample}

Our study consisted of 651 participants aged $\nsucceq 60$ years who were enrolled in the ALSTAR study. Characteristics of this cohort have been previously described. ${ }^{45,46}$ In brief, participants with normal macular health and early to intermediate AMD in the absence of other retinal diseases were recruited from 2 primary care ophthalmology practices in the Callahan Eye Hospital at the University of Alabama at Birmingham. The presence and severity of AMD were assessed at enrollment using digital color fundus photos (Zeiss FF 450plus fundus camera, Carl Zeiss Meditec, Inc., Dublin, CA) and evaluated by a certified grader (M.E.C.) using the Age-Related Eye Disease Study (AREDS) 9-step severity scale. ${ }^{49}$ Grade 1 represents normal macular health, grades 2 to 4 represent early AMD, and grades 5 to 8 represent intermediate AMD. Following the AREDS protocol, ${ }^{50}$ the presence of "reticular drusen" was recorded as present by the same grader when characteristic patterns were detected within the CFP. Basic demographics, as well as information about smoking status, alcohol use, and medical history were collected at the baseline visit using intervieweradministered questionnaires as described. ${ }^{45}$ Plasma concentrations of apolipoprotein (apo) $\mathrm{B}$ and apo A-I, complement proteins (C3, C4, C5), complement protein fragments (C3a, $\mathrm{C} 4 \mathrm{a}, \mathrm{C} 5 \mathrm{a}$ ), and C-reactive protein (CRP) were assessed at the baseline visit as described. ${ }^{45}$ 


\section{Study Procedures}

Imaging modalities-SD-OCT images were obtained with Spectralis HRA + SD-OCT (Heidelberg Engineering, Heidelberg, Germany). Macula volumes were centered over the fovea with horizontal scans across an area of $20^{\circ} \times 15^{\circ}(5.7 \times 4.3 \mathrm{~mm})$. Optic nerve head $(\mathrm{ONH})$ volumes were centered over the $\mathrm{ONH}$ within a $20^{\circ}(5.8 \pm 0.1 \mathrm{~mm})$ diameter area, with radial scans. The IR $(\lambda=830 \mathrm{~nm})$ and FAF (excitation, $488 \mathrm{~nm}$; emission, $>600 \mathrm{~nm})$ images were acquired with the confocal scanning laser ophthalmoscope (Spectralis HRA + SDOCT; Heidelberg Engineering, Heidelberg, Germany). Fields of view of $30^{\circ} \times 30^{\circ}$ were digitized at $1536 \times 1536$ pixels and centered over the macula and ONH for IR images, and centered over the macula for FAF images. These images were analyzed with Spectralis software that allowed spatial point-to-point correlation between SD-OCT and IR (Heidelberg Eye Explorer, HEYEX version 1.6.4.0 with Spectralis Viewing Module 5.3.2.0; Heidelberg Engineering, Heidelberg, Germany). Color digital $30^{\circ}$ fundus photographs that were centered over the $\mathrm{ONH}$, macula, and temporal to macula were taken with a FF450 Plus fundus camera (Carl Zeiss Meditec, Inc., Dublin, CA) after pupil dilation.

SD-OCT was considered gradable if: 1) it included both the macular volume with 73 horizontal B-scans at $60 \mu \mathrm{m}$ intervals and the $\mathrm{ONH}$ volume with 48 radial B-scans; 2) the grader could distinguish the area between (and inclusive of) external limiting membrane and RPE-Bruch's membrane complex on both volumes; 3) SDD were present and discernable in any of the available B-scans (even if not $100 \%$ of the total number of scans). En-face images (IR, FAF, CFP) were considered gradable if there was 1 image that was sufficiently focused to reveal fine vessels and subtle detail in either color or monochrome as appropriate. (see Definitions).

The detection of SDD was performed by 1 masked grader (A.V.Z., an ophthalmologist), with an intragrader agreement of 0.73 . SD-OCT and IR images were compared point-to-point using the Spectralis optical coherence tomography (OCT) mapping software. Lesions on FAF images were indirectly correlated with SD-OCT via IR images using retinal vasculature as landmarks. The area outside the SD-OCT volume but within the area delimited by FAF and IR imaging was similarly examined for specific features of SDD. CFP also were examined again at this time, independently of the initial AREDS grading of reticular drusen described earlier. Characteristic subretinal reflectivity could be confirmed for lesions within macula and ONH SD-OCT volumes, and the grader learned through practice with this dataset to recognize out-of-volume lesions that were visible only in en-face images.

Definitions-The SDD in the ALSTAR cohort were less abundant overall than in cohorts with many patients with AMD, and thus definitions relying solely on the pattern made by a group of lesions were not applicable. ${ }^{47}$ We developed definitions for the presence of SDD at image, eye, and person levels (Supplementary Table 2, available at http:// www.aaojournal.org). We used the image-level definition to determine the presence of SDD on individual imaging modalities. The eye-level definition was used to determine SDD presence in individual eyes. Finally, the person-level definition was used for calculation of SDD prevalence. 


\section{Image level}

Subretinal drusenoid deposits were considered Present and Discernable when at least 1 of listed criteria was met for individual modalities:

SD-OCT: One or more dome-shaped or oval hyper-reflective material of $\geq 25 \mu \mathrm{m}$ in size internal to and adjacent to the RPE with or without various degrees of disturbance of the ellipsoid zone. ${ }^{7,}{ }^{15}$ IR: typical multiple discrete or interlacing hyporeflective foci (dot-, ribbon-shaped or spots) ${ }^{30}$ of $\geq 25 \mu \mathrm{m}$ in size and grouped in an arcuate pattern along the superotemporal arcades or perifovea superior and nasal to macula and $\mathrm{ONH}$, may be illdefined; discrete solitary hyporeflective foci (dot-, oval-shaped or spots) of $\geq 25 \mu \mathrm{m}$ in size correlating with SDD in the same location within macular and ONH SD-OCT volumes; ${ }^{33}$ discrete dots of any size with an iso- or hyper-reflective center surrounded by hyporeflective ring (target). ${ }^{51}$ FAF: typical multiple discrete or interlacing hypoautofluorescent foci against a mildly elevated AF background and grouped in an arcuate pattern along the superotemporal arcades or perifovea superior and nasal to macula and $\mathrm{ONH}$, may be illdefined; discrete solitary hypoautofluorescent foci correlating with SDD on IR in the same location within macular and ONH SD-OCT volumes; ${ }^{33}$ discrete dots of any size with isoautofluorescent center surrounded by a ring of reduced autofluorescence (target). ${ }^{51} \mathrm{CFP}$ : typical multiple light yellowish-pale or grayish discrete dots/globules or interlacing ribbons ${ }^{30}$ grouped in an arcuate pattern along the superotemporal arcades or perifovea superior and nasal to macula and ONH that appear slightly whiter or grayer than soft drusen. This CFP criterion was defined independently of the AREDS scale.

Subretinal drusenoid deposits were considered Questionable when at least 1 of listed criteria was met for individual modalities:

SD-OCT: local thickening of RPE or dot-like subretinal reflectivity with intact ellipsoid zone; various-degree disturbances of ellipsoid zone without evidence of hyper-reflective subretinal deposits. ${ }^{52} I R$ : atypical hyporeflective clusters or suspicious solitary hyporeflective discrete foci located outside the SD-OCT volume. FAF: atypical hypoautofluorescent clusters or suspicious solitary hypoautofluorescent discrete foci located outside the SD-OCT volume. CFP: suspicious solitary yellowish-pale or grayish discrete foci; atypical clusters of yellowish/grayish discrete foci that could not be distinguished on a background.

Subretinal drusenoid deposits were considered Not Discernable when there were no visible features of SDD on any imaging modality.

Subretinal drusenoid deposits were considered Ungradable if suboptimal image quality prevented the grader from making any of the above judgments; in an incomplete SD-OCT volume, no SDD or questionable SDD only are present.

\section{Eye level}

To estimate SDD presence at the eye level we developed 2 sets of criteria: strict and expanded. To fulfill strict criteria, a patient was required to have SDD judged as present and discernable on SD-OCT and at least 1 en-face modality; or SDD judged as present and 
discernable on 2 en-face modalities in the absence of SD-OCT findings. To fulfill expanded criteria, a patient was required to have SDD judged as present and discernable or questionable on any image modality. Features of SDD demonstrated on different modalities used are shown in Figure 1. An example of a solitary macular SDD in a non-AMD participant is shown in Figure 2. Examples of SDD and basal laminar deposit in the peripapillary region are shown in Figure 3.

\section{Person level}

To report SDD prevalence at the person level, we considered SDD present if it was detected in 1 or both eyes. SDD prevalence at the person-level was obtained for the whole sample and its subgroups stratified by AMD status and severity. Continuous and categorical factors were compared between those with and without SDD using t-tests and chi-square tests, respectively. Logistic regression was used to calculate age-adjusted odds ratios (ORs) and 95\% confidence intervals (CIs) between SDD status and presence and severity of AMD. The number of subjects excluded from the final analysis varied because of different gradability requirements for SD-OCT images between strict and expanded criteria at the person-level.

\section{Results}

A total of 651 participants were enrolled in the study. After excluding 35 participants because of incomplete imaging data and 5 with eye conditions that did not meet the inclusion criteria, 611 participants were available for analysis. Table 3 shows basic demographic, lifestyle characteristics and medical results stratified by SDD status using strict criteria at the person level. Overall, the mean age of participants was 69 years (60-92 years), $65 \%$ were females, and $95 \%$ were whites. Non-smokers comprised $54 \%$ of the group. By self-report, one third of participants had heart disease, and half were hypertensive. Agerelated macular degeneration was present in 32\% of participants. Of those with AMD, $90 \%$ had early AMD (AREDS 2-4) and the remainder had intermediate AMD (AREDS 5-8). Table 4 shows SDD presence at the person-level using strict criteria, stratified by AMD status (AREDS 1-8).

By using strict criteria, the prevalence of SDD in the overall sample was $32 \%$ (197/611). Of the 197 persons with SDD, 122 (62\%) were affected in both eyes. Participants with SDD were significantly older than those without SDD (70.6 vs. 68.7 years, $\mathrm{p}=0.0002)$. In subjects with normal macular health, SDD prevalence was $23 \%$ (95/413). In subjects with AMD, SDD were present in 52\% (101/194) ( $\mathrm{p}<0.0001)$. The prevalence of SDD correlated with severity of AMD; among those with early and intermediate AMD, SDD prevalence was $49 \%$ and $79 \%$, respectively $(\mathrm{p}<0.0001)$. After age adjustment, those with SDD were 3.4 times more likely to have AMD than those without SDD (95\% CI 2.3-4.9). No statistically significant association was found between SDD and gender, race, level of education, high blood pressure, heart disease, smoking, alcohol consumption, and statin use. None of the analyzed serologic tests including apolipoprotein B and A-I, complement proteins (C3, C4, C5), complement protein fragments (C3a, C4a, C5a), and CRP were associated with SDD presence. Supplementary Table 5 shows serologic results stratified by SDD status using strict criteria at the person level (available at http://www.aaojournal.org). 
By expanded criteria, SDD prevalence in the overall sample was $74 \%$. Seventy percent of those with SDD were affected in both eyes. The AMD status and severity remained statistically significant predictors of SDD, whereas age, along with other demographic, medical and serologic variables, were not associated with SDD presence. After ageadjustment, persons with SDD were 2.5 times more likely to have AMD than those without SDD (95\% CI 1.6-4.0).

We assessed whether the association between age and SDD is affected by AMD severity and whether the association between AMD severity and SDD is modified by age. The ageadjusted ORs were not meaningfully different from the crude ORs suggesting minimal confounding with age. Table 6 summarizes prevalence of SDD and crude and adjusted associations by age and AMD presence and severity using strict and expanded criteria at the person level.

To compare our results with those of population-based studies, we limited our analysis to SDD detected on CFP by AREDS criteria. ${ }^{50}$ The resulting SDD prevalence was $1.97 \%$ $(12 / 610)$ at the person level and $1.48 \%(9 / 608)$ at the eye level (with worse acuity as a metric).

We then assessed the impact of ascertainment area on the person level prevalence by determining the distribution of SDD in macula and ONH OCT volumes in the overall sample and among persons with SDD. This analysis included the overall sample of 611 persons and a sample of 190 persons with SDD detected on SD-OCT and confirmed by at least 1 en-face modality. Referenced to all 611 persons in the sample, $101(16.5 \%)$ and 185 (30.3\%) persons had at least one present and discernable SDD in macula and ONH volumes, respectively. Among the 190 persons with SDD confirmed by SD-OCT, $2.6 \%(5 / 190)$ exhibited SDD in macula SD-OCT volumes alone, 46.8\% (89/190) exhibited SDD in ONH SD-OCT volumes alone, and 50.5\% (96/190) in both volumes. Thus, almost twice as many persons had SDD in the ONH volume than in the macula volume.

\section{Discussion}

Our major finding is a $23 \%$ SDD prevalence in subjects with maculas considered healthy by CFP, higher than previously reported for study populations with many normal subjects. ${ }^{21,44}$ Another major finding was that SDD were frequent in subjects with early or intermediate AMD (49\% and 79\%, respectively). The prevalence of SDD correlated with age and AMD presence and severity. Reminiscent of the Beaver Dam Eye Study (BDES) reporting $\geq 1$ macular drusen in $95.5 \%$ of the population $>45$ year on the basis of validated CFP grading ${ }^{55}$, a quarter century later we find that many eyes $\nsucceq 60$ years considered normal by CFP also have $\geq 1$ SDD on the basis of multimodal imaging. To appreciate the significance of our findings, we discuss several important factors affecting SDD prevalence.

\section{Subretinal drusenoid deposit visibility and definition}

Most experts consider SDD present when lesions form a pattern on various en-face imaging modalities, with SD-OCT used to confirm, ${ }^{56-58}$ whereas others use point-to-point correlation of discrete lesions on different modalities. ${ }^{14,53,54}$ Histologically, SDD were 
diagnosed when at least 3 lesions were present in 2 standard sections of any 1 eye, ${ }^{1}$ and individual lesions with width of 8-17 $\mu \mathrm{m}$ have typical SDD fine structure. ${ }^{1}$ In consideration of these findings, we included small sparse lesions and differentiated them from drusen, which is difficult using CFP alone, by using a multimodal approach.. This strategy also allowed us to avoid overestimation of SDD prevalence by our expanded criteria. Another important consideration in estimating SDD prevalence is the denominator used for statistical calculations. Because SDD are mostly bilateral, ${ }^{21,35,58}$ SDD occurrence at the eye level is likely to overestimate true prevalence. Therefore, we reported SDD prevalence at the person level.

In normal eyes and eyes with early AMD, SDD are often subtle, smaller, and certainly less numerous than in eyes with intermediate and advanced AMD. Whereas existing descriptions focused on patterns over extensive areas, we saw many single and sparse lesions (i.e., not forming a pattern). Literature to date has debated whether SDD lesions fully account for the clinical appearance of reticular pseudodrusen (against: ${ }^{52}$; for: ${ }^{1,7,15,59}$ ) with recent evidence tipping towards equivalence of the two. A second question is whether SDD align with choroidal structure, and evidence is tipping towards non-alignment. ${ }^{60}$ Technical challenges to answering these questions are also relevant to our current study. These include inaccuracies in point-to-point alignment of the Spectralis, ${ }^{61}$ the presence of many small repeating elements in outer retinal layers, and the dual requirements of comprehensive imaging and appropriate statistics to establish specificity and sensitivity of overlap with other anatomical features. Without attention to these factors it is possible to get spurious alignments. ${ }^{62,63}$ Biological challenges include a morphological heterogeneity that may reflect different stages of a lesion lifecycle. ${ }^{30}$ Our study shows that it is possible to crossvalidate SD-OCT and en-face images for sparse subretinal lesions. Although lesions that fall between scans can be missed, verifying the identities of lesions that are found can be unambiguous. Our task in normal and early AMD eyes is thus easier in this respect than in eyes with more advanced disease.

The ALSTAR study, originated in 2009, was not designed to find SDD. Current technology is limiting in any case. In normal eyes, SDD tend to appear first outside the macular OCT volumes and therefore cannot be verified with SD-OCT. However, after the grader studied SDD in macula and ONH SD-OCT volumes where confirmation of subretinal material was possible, out-of-volume SDD could be recognized confidently.

\section{Populations studied}

By using strict criteria, we detected SDD in $32 \%$ of participants of the ALSTAR cohort. Two thirds of subjects had normal maculas and one third had predominantly early AMD. Our results cannot be directly compared to other clinic-based studies that use retina clinic populations because we recruited our participants from primary eye care clinics. Even among studies based in retina clinics, prevalence estimates vary widely. In these previous studies, AMD presence and severity appear to affect SDD prevalence, which increases from $3.0 \%$ to $93.6 \%$ when subjects with late AMD are included in analyses. $16,28,53$ When we limit the comparison of clinic-based studies to only those subjects with early AMD, our 49\% SDD prevalence at the person-level based on strict criteria is higher than $31.5 \%$ prevalence 
at the eye level reported by De Bats et al. ${ }^{53}$ Likewise, $79 \%$ of our participants with intermediate AMD had SDD by strict criteria, compared with 30\% of SDD prevalence at the person level reported by Wu et al. ${ }^{54}$ Differences are likely explained by these authors' restrictive SDD definition, which required at least 5 subretinal lesions, fewer B-scans ( 3153 and $49^{54}$ ) and absence of an ONH volume. In contrast, in our study ONH SD-OCT volumes were instrumental in detecting lesions in almost half of all persons with SDD by strict criteria. These results indicate that the wider area of SDD ascertainment was a major determinant of the high SDD prevalence in our study, and that the ONH region has a greater SDD involvement than the macula in the same eye.

To compare and contextualize our results from multimodal imaging with those from population-based studies, we assessed SDD as assessed by the AREDS study in CFP alone. By using SDD presence in the eye with worse acuity as a metric, SDD prevalence in the ALSTAR cohort was $1.48 \%$, compared with $0.7 \%$ in Beaver Dam Eye Study. ${ }^{21}$ Using SDD presence in either eye as a metric, SDD prevalence in the ALSTAR cohort was $1.97 \%$, compared to $1.95 \%$ in Blue Mountain Eye Study (BMES). ${ }^{44}$ Thus, our SDD prevalence estimates are similar to those obtained in population-based studies when CFP imaging and similar metrics are used. When determined from multimodal imaging including OCT volumes of macula and $\mathrm{ONH}$, SDD prevalence in our predominantly normal cohort, even with strict criteria, is approximately 10-fold higher than in the BDES or BMES and is comparable to some studies of AMD patients in retina clinics (Supplementary Table 1, available at http://www.aaojournal.org). We considered 4 possible explanations for this result: 1) the ALSTAR cohort ( $>60$ year) is at the older end of the BDES and BMES agerange ( $>43$ or $>45$ year); 2 ) we used multimodal imaging including SD-OCT volumes, enabling detection of subtle subretinal lesions; 3 ) we counted solitary and sparse lesions, in addition to numerous lesions in a distinctive pattern, a criterion common to all prior population-based studies; ${ }^{21,44}$ and 4 ) we examined both macula and peripapillary areas, whereas many studies including BDES and BMES examined macula only.

Thus, the prevalence of SDD is dramatically affected by SDD definition, imaging modality, ascertainment area, person- versus eye-level calculations, and patient population. Despite methodological differences, strong associations of SDD with age and AMD presence and severity were detected, in concordance with other studies. ${ }^{14,44,56,64}$

\section{Subretinal drusenoid deposits, age, and age-related macular degeneration}

We found that octogenarians are 4 times more likely to have SDD compared to sexagenarians. High prevalence of SDD among participants with healthy maculas in association with older age raises the possibility that SDD simply may manifest normal aging in the outer retinal neurovascular unit (photoreceptors, ${ }^{65-67}$ RPE, ${ }^{68,69}$ Bruch's membrane, ${ }^{5}$ and choroid ${ }^{70-72}$ ). This notion is supported by our recent findings (Neely, D., et al. Association between subretinal drusenoid deposits seen by multimodal imaging and dark adaptation in normal, early, and intermediate age-related macular degeneration eyes. Invest Ophthalmol Vis Sci 2015; 56(7): 2777. ${ }^{10}$ ) that in the absence of AMD, SDD do not affect rod-mediated dark adaptation, a functional test that is affected by disease more than by aging. ${ }^{46}$ However, SDD are strongly associated with AMD in the current analysis: presence 
of SDD increased the odds of early and intermediate AMD diagnosis 3- and 11-fold, respectively. It is possible that only certain SDD phenotypes predispose eyes to AMD. However, because this study is cross-sectional, future studies focusing on the temporal coevolution of SDD and AMD are needed to determine true pathogenic significance of SDD.

\section{Risk factors for subretinal drusenoid deposits}

Similar to some studies ${ }^{14,56}$ and unlike others, ${ }^{21,22,35}$ the current study did not find an association between SDD and female sex, smoking, hypertension, and cardiovascular disease. Also, we did not find a correlation between SDD and factors previously associated (systemic complement components and CRP ${ }^{73-75}$ ) or not associated with AMD (plasma apolipoprotein B and A-I). ${ }^{76}$ This suggests that although SDD are associated with incidence and development of late ${ }^{21,28,77}$ as well as early AMD (Huisingh, C., et al. The association between SDD in older adults in normal macular health and incident AMD, IOVS, Accepted for publication), they may not share the same pathophysiology. The formation of SDD behind the blood-retina barrier in the subretinal space may be relatively shielded from systemic factors and instead influenced by local metabolic abnormalities, such as impaired lipid cycling in rod-dominant areas. ${ }^{1,78}$

In this regard, our findings importantly solidify the relationship of SDD with rod photoreceptors by demonstrating their similar topographies. Rods are most numerous in a horizontally oriented elliptical ring, cresting at the edge of the macula and surrounding the fovea and ONH (Supplementary Figure 4, available at www.aaojournal.org), ${ }^{79}$ strikingly similar to the distribution of SDD. ${ }^{35}$ Further, SDD is 2-fold more prevalent in $\mathrm{ONH}$ volumes than in the macula. By knowing $\mathrm{ONH}$ dimensions ${ }^{80}$ and the specifications of the Spectralis, we can verify that SDD abundance near the ONH is not explained by a higher sampling density of A-scans, because ONH sampling density is approximately $50 \%$ less than that of the macula. Thus, higher SDD prevalence near the $\mathrm{ONH}$ is due to more numerous or larger lesions that affect the probability of detection, in addition to an unknown amount of peripapillary basal laminar deposits (Figure 3), which are prominent in older eyes. ${ }^{81}$ The differential distribution of SDD (perifoveal and peripapillary regions) and basal linear deposit/soft drusen (central macula) is hypothesized to reflect differential requirements of cholesterol homeostasis in rod and cone photoreceptors, respectively. ${ }^{1}$

\section{Study strengths and limitations}

Strengths of this study include a relatively large number of participants in normal macular health from ophthalmology primary care clinics. By including sparse lesions and lesions in patterns, and correlating them point-to-point in multimodal imaging including SD-OCT, we solidified a definition of SDD for use in future research. The fact that so many lesions were not part of a reticular pattern helps reinforce the appropriateness of the SDD terminology, because "reticular" may be just one stage in the lesion life cycle. ${ }^{30}$ Another strength was the novel inclusion of SD-OCT ONH volumes for screening peripapillary retina, where solitary SDD were common (Steinberg et al ${ }^{48}$ ). This study also has limitations addressable in future research. Subretinal drusenoid deposits beyond the arcades and ONH is outside the typical scan volumes of current SD-OCT, and in the future, detection will be facilitated by widefield imaging. ${ }^{82}$ As in all studies of this nature, we do not have histology of graded eyes, but 
we did have access to histology demonstrating the existence of sparse lesions. We relied on grader learning and intra-grader reliability in the absence of direct histologic validation, a strategy used by early epidemiology studies based on CFP. Finally, this study is a clinicbased study in that it recruited patients from 2 primary eye care clinics. To what extent our SDD prevalence estimates apply to the general population of persons in normal macular health or with early AMD remains to be determined.

In conclusion, when systematically assessed with multimodal imaging over an ascertainment area including macula, $\mathrm{ONH}$ volumes, and out-of-volume retina seen in en-face imaging, SDD are more prevalent in the elderly than previously reported (23\% of persons $>60$ years of age). Other published findings, have shown that SDD is a risk factor for AMD advancement, independently of drusen. Longitudinal studies 5 to 20 years in duration have proven drusen very important for AMD progression. Because SDD and drusen are plausibly related in outer retinal biology, SDD merit our attention. Finally, multimodal imaging is likely to be implemented in the clinic routinely in the future, and therefore more ophthalmologists will see SDD. Consensus on SDD definition and newer imaging tools for screening will improve accuracy of SDD diagnosis and enhance our understanding of clinical and biological SDD significance.

\section{Supplementary Material}

Refer to Web version on PubMed Central for supplementary material.

\section{Acknowledgments}

Financial Support: National Institute on Aging (R01AG04212) and National Eye Institute (R01EY06109, R01EY024378), National Institutes of Health, Bethesda MD; EyeSight Foundation of Alabama, Birmingham AL; Alfreda J. Schueler Trust, Chicago IL; Research to Prevent Blindness Inc., New York NY.

\section{Abbreviations and Acronyms}

$\begin{array}{ll}\text { ALSTAR } & \text { The Alabama Study of Early Age-Related Macular Degeneration } \\ \text { AMD } & \text { Age-related macular degeneration } \\ \text { APO A-I } & \text { Apolipoprotein A-I } \\ \text { APO B } & \text { Apolipoprotein B } \\ \text { AREDS } & \text { Age-Related Eye Disease Study } \\ \text { BDES } & \text { Beaver Dam Eye Study } \\ \text { BMES } & \text { Blue Mountain Eye Study } \\ \text { BR } & \text { Blue reflectance (488 nm excitation) } \\ \text { CFP } & \text { color fundus photographs } \\ \text { CI } & \text { Confidence interval } \\ \text { CNV } & \text { Choroidal neovascular membrane } \\ \text { CRP } & \text { C-reactive protein }\end{array}$




\begin{tabular}{|c|c|}
\hline cSLO & Confocal scanning laser ophthalmoscope \\
\hline C3-5 & Complement proteins $\mathrm{C} 3, \mathrm{C} 4, \mathrm{C} 5$ \\
\hline C3a-5a & Complement protein fragments $\mathrm{C} 3 \mathrm{a}, \mathrm{C} 4 \mathrm{a}, \mathrm{C} 5 \mathrm{a}$ \\
\hline $\mathbf{E L}$ & Eye-level \\
\hline FA & fluorescein angiography \\
\hline FAF & Fundus autofluorescence \\
\hline FAF-NIR & Fundus autofluorescence, near infrared ( $830 \mathrm{~nm}$ excitation) \\
\hline FAF-SW & Fundus autofluorescence, short wavelength (488 nm excitation) \\
\hline GA & Geographic atrophy \\
\hline IR & Infrared reflectance \\
\hline ICG & Indocyanine green angiography \\
\hline ND & Not Discernable \\
\hline NIA & Near-infrared autofluorescence (787 nm excitation) \\
\hline NIR-R & Near infrared reflectance (820 $\mathrm{nm}$ excitation) \\
\hline NIR-FAF & Near infrared reflectance, fundus autofluorescence (790 nm excitation) \\
\hline OCT & Optical coherence tomography \\
\hline $\mathbf{O R}$ & Odds ratio \\
\hline ONH & Optic nerve head \\
\hline PAD & Present and Discernable \\
\hline PL & Person-level \\
\hline $\mathbf{Q}$ & Questionable \\
\hline RPD & Reticular pseudodrusen \\
\hline Ref & Reference \\
\hline $\mathbf{R F}$ & Red-free light \\
\hline RPE & Retinal pigment epithelium \\
\hline SDD & Subretinal drusenoid deposits \\
\hline SD-OCT & Spectral domain optical coherence tomography \\
\hline $\mathbf{U}$ & Ungradable \\
\hline
\end{tabular}

\section{References}

1. Curcio CA, Messinger JD, Sloan KR, et al. Subretinal drusenoid deposits in non-neovascular agerelated macular degeneration: morphology, prevalence, topography, and biogenesis model. Retina. 2013; 33(2):265-76. [PubMed: 23266879]

Ophthalmology. Author manuscript; available in PMC 2017 May 01. 
2. Schmitz-Valckenberg S, Alten F, Steinberg JS, et al. Reticular drusen associated with geographic atrophy in age-related macular degeneration. Invest Ophthalmol Vis Sci. 2011; 52(9):5009-15. [PubMed: 21498612]

3. Rudolf M, Malek G, Messinger JD, et al. Sub-retinal drusenoid deposits in human retina: organization and composition. Exp Eye Res. 2008; 87(5):402-8. [PubMed: 18721807]

4. Oak AS, Messinger JD, Curcio CA. Subretinal drusenoid deposits: further characterization by lipid histochemistry. Retina. 2014; 34(4):825-6. [PubMed: 24589874]

5. Curcio CA, Johnson M, Rudolf M, Huang JD. The oil spill in ageing Bruch membrane. Br J Ophthalmol. 2011; 95(12):1638-45. [PubMed: 21890786]

6. Arnold JJ, Sarks JP, Killingsworth MC, et al. Adult vitelliform macular degeneration: a clinicopathological study. Eye (Lond). 2003; 17(6):717-26. [PubMed: 12928683]

7. Zhang Y, Wang X, Rivero EB, et al. Photoreceptor perturbation around subretinal drusenoid deposits as revealed by adaptive optics scanning laser ophthalmoscopy. Am J Ophthalmol. 2014; 158(3): 584-96 e1. [PubMed: 24907433]

8. Steinberg JS, Fitzke FW, Fimmers R, et al. Scotopic and Photopic Microperimetry in Patients With Reticular Drusen and Age-Related Macular Degeneration. JAMA Ophthalmol. 2015

9. Flamendorf J, Agron E, Wong WT, et al. Impairments in Dark Adaptation Are Associated with AgeRelated Macular Degeneration Severity and Reticular Pseudodrusen. Ophthalmology. 2015; 122(10):2053-62. [PubMed: 26253372]

10. Neely DZ, AV, Clark ME, Huisingh CE, Jackson GR, McGwin G, Curcio CA, Owsley C. Association between subretinal drusenoid deposits seen by multimodal imaging and dark adaptation in normal, early, and intermediate age-related macular degeneration eyes. Abstract. Invest Ophthalmol Vis Sci. 2015; 56(7):2777.

11. Mimoun G, Soubrane G, Coscas G. Macular drusen. J Fr Ophtalmol. 1990; 13(10):511-30. [PubMed: 2081842]

12. Arnold JJ, Sarks SH, Killingsworth MC, Sarks JP. Reticular pseudodrusen. A risk factor in agerelated maculopathy. Retina. 1995; 15(3):183-91. [PubMed: 7569344]

13. Curcio CA, Presley JB, Malek G, et al. Esterified and unesterified cholesterol in drusen and basal deposits of eyes with age-related maculopathy. Exp Eye Res. 2005; 81(6):731-41. [PubMed: 16005869]

14. Zweifel SA, Imamura Y, Spaide TC, et al. Prevalence and significance of subretinal drusenoid deposits (reticular pseudodrusen) in age-related macular degeneration. Ophthalmology. 2010; 117(9):1775-81. [PubMed: 20472293]

15. Zweifel SA, Spaide RF, Curcio CA, et al. Reticular pseudodrusen are subretinal drusenoid deposits. Ophthalmology. 2010; 117(2):303-12 e1. [PubMed: 19815280]

16. Prenner JL, Rosenblatt BJ, Tolentino MJ, et al. Risk factors for choroidal neovascularization and vision loss in the fellow eye study of CNVPT. Retina. 2003; 23(3):307-14. [PubMed: 12824829]

17. Einbock W, Moessner A, Schnurrbusch UE, et al. Changes in fundus autofluorescence in patients with age-related maculopathy. Correlation to visual function: a prospective study. Graefes Arch Clin Exp Ophthalmol. 2005; 243(4):300-5. [PubMed: 15864618]

18. Smith RT, Chan JK, Busuoic M, et al. Autofluorescence characteristics of early, atrophic, and highrisk fellow eyes in age-related macular degeneration. Invest Ophthalmol Vis Sci. 2006; 47(12): 5495-504. [PubMed: 17122141]

19. Cohen SY, Dubois L, Tadayoni R, et al. Prevalence of reticular pseudodrusen in age-related macular degeneration with newly diagnosed choroidal neovascularisation. Br J Ophthalmol. 2007; 91(3):354-9. [PubMed: 16973663]

20. Wang JJ, Rochtchina E, Lee AJ, et al. Ten-year incidence and progression of age-related maculopathy: the blue Mountains Eye Study. Ophthalmology. 2007; 114(1):92-8. [PubMed: 17198852]

21. Klein R, Meuer SM, Knudtson MD, et al. The epidemiology of retinal reticular drusen. Am J Ophthalmol. 2008; 145(2):317-26. [PubMed: 18045568]

22. Smith RT, Merriam JE, Sohrab MA, et al. Complement factor $\mathrm{H} 402 \mathrm{H}$ variant and reticular macular disease. Arch Ophthalmol. 2011; 129(8):1061-6. [PubMed: 21825189] 
23. Smailhodzic D, Fleckenstein M, Theelen T, et al. Central areolar choroidal dystrophy (CACD) and age-related macular degeneration (AMD): differentiating characteristics in multimodal imaging. Invest Ophthalmol Vis Sci. 2011; 52(12):8908-18. [PubMed: 22003107]

24. Forte R, Querques G, Querques L, et al. Multimodal imaging of dry age-related macular degeneration. Acta Ophthalmol. 2012; 90(4):e281-7. [PubMed: 22269083]

25. Alten F, Clemens CR, Milojcic C, Eter N. Subretinal drusenoid deposits associated with pigment epithelium detachment in age-related macular degeneration. Retina. 2012; 32(9):1727-32. [PubMed: 22466490]

26. Ueda-Arakawa N, Ooto S, Tsujikawa A, et al. Sensitivity and specificity of detecting reticular pseudodrusen in multimodal imaging in Japanese patients. Retina. 2013; 33(3):490-7. [PubMed: 23403515]

27. Xu L, Blonska AM, Pumariega NM, et al. Reticular macular disease is associated with multilobular geographic atrophy in age-related macular degeneration. Retina. 2013; 33(9):1850-62. [PubMed: 23632954]

28. Marsiglia M, Boddu S, Bearelly S, et al. Association between geographic atrophy progression and reticular pseudodrusen in eyes with dry age-related macular degeneration. Invest Ophthalmol Vis Sci. 2013; 54(12):7362-9. [PubMed: 24114542]

29. Alten F, Clemens CR, Heiduschka P, Eter N. Characterisation of reticular pseudodrusen and their central target aspect in multi-spectral, confocal scanning laser ophthalmoscopy. Graefes Arch Clin Exp Ophthalmol. 2014; 252(5):715-21. [PubMed: 24276561]

30. Suzuki M, Sato T, Spaide RF. Pseudodrusen subtypes as delineated by multimodal imaging of the fundus. Am J Ophthalmol. 2014; 157(5):1005-12. [PubMed: 24503406]

31. Lee MY, Ham DI. Subretinal drusenoid deposits with increased autofluorescence in eyes with reticular pseudodrusen. Retina. 2014; 34(1):69-76. [PubMed: 23743636]

32. Saade C, Smith RT. Reticular macular lesions: a review of the phenotypic hallmarks and their clinical significance. Clin Experiment Ophthalmol. 2014; 42(9):865-74. [PubMed: 24803342]

33. Spaide RF, Curcio CA. Drusen characterization with multimodal imaging. Retina. 2010; 30(9): 1441-54. [PubMed: 20924263]

34. Steinberg JS, Gobel AP, Fleckenstein M, et al. Reticular drusen in eyes with high-risk characteristics for progression to late-stage age-related macular degeneration. Br J Ophthalmol. 2015

35. Ueda-Arakawa N, Ooto S, Nakata I, et al. Prevalence and genomic association of reticular pseudodrusen in age-related macular degeneration. Am J Ophthalmol. 2013; 155(2):260-9 e2. [PubMed: 23111182]

36. Kim JH, Chang YS, Kim JW, et al. Prevalence of Subtypes of Reticular Pseudodrusen in Newly Diagnosed Exudative Age-Related Macular Degeneration and Polypoidal Choroidal Vasculopathy in Korean Patients. Retina. 2015

37. De Bats F, Wolff B, Mauget-Faysse M, et al. Association of reticular pseudodrusen and early onset drusen. ISRN Ophthalmol. 2013; 2013:273085. [PubMed: 24563787]

38. Zweifel SA, Maygar I, Berger W, et al. Multimodal imaging of autosomal dominant drusen. Klin Monbl Augenheilkd. 2012; 229(4):399-402. [PubMed: 22496012]

39. Gliem M, Hendig D, Finger RP, et al. Reticular pseudodrusen associated with a diseased bruch membrane in pseudoxanthoma elasticum. JAMA Ophthalmol. 2015; 133(5):581-8. [PubMed: 25764262]

40. Gliem M, Muller PL, Mangold E, et al. Reticular Pseudodrusen in Sorsby Fundus Dystrophy. Ophthalmology. 2015

41. Lally DR, Baumal C. Subretinal drusenoid deposits associated with complement-mediated IgA nephropathy. JAMA Ophthalmol. 2014; 132(6):775-7. [PubMed: 24921170]

42. Aleman TS, Garrity ST, Brucker AJ. Retinal structure in vitamin A deficiency as explored with multimodal imaging. Doc Ophthalmol. 2013; 127(3):239-43. [PubMed: 23900584]

43. Genead MA, Fishman GA, Lindeman M. Spectral-domain optical coherence tomography and fundus autofluorescence characteristics in patients with fundus albipunctatus and retinitis punctata albescens. Ophthalmic Genet. 2010; 31(2):66-72. [PubMed: 20450307] 
44. Joachim N, Mitchell P, Rochtchina E, et al. Incidence and progression of reticular drusen in agerelated macular degeneration: findings from an older Australian cohort. Ophthalmology. 2014; 121(4):917-25. [PubMed: 24332537]

45. Owsley C, Huisingh C, Jackson GR, et al. Associations between abnormal rod-mediated dark adaptation and health and functioning in older adults with normal macular health. Invest Ophthalmol Vis Sci. 2014; 55(8):4776-89. [PubMed: 24854857]

46. Owsley C, Huisingh C, Clark ME, et al. Comparison of Visual Function in Older Eyes in the Earliest Stages of Age-related Macular Degeneration to Those in Normal Macular Health. Curr Eye Res. 2015:1-7.

47. Sarks J, Arnold J, Ho IV, et al. Evolution of reticular pseudodrusen. Br J Ophthalmol. 2011; 95(7): 979-85. [PubMed: 21109695]

48. Steinberg JS, Auge J, Jaffe GJ, et al. Longitudinal analysis of reticular drusen associated with geographic atrophy in age-related macular degeneration. Invest Ophthalmol Vis Sci. 2013; 54(6): 4054-60. [PubMed: 23633663]

49. Davis MD, Gangnon RE, Lee LY, et al. The Age-Related Eye Disease Study severity scale for agerelated macular degeneration: AREDS Report No. 17. Arch Ophthalmol. 2005; 123(11):1484-98. [PubMed: 16286610]

50. Age-Related Eye Disease Study Research G. The Age-Related Eye Disease Study system for classifying age-related macular degeneration from stereoscopic color fundus photographs: the Age-Related Eye Disease Study Report Number 6. Am J Ophthalmol. 2001; 132(5):668-81. [PubMed: 11704028]

51. Querques G, Querques L, Martinelli D, et al. Pathologic insights from integrated imaging of reticular pseudodrusen in age-related macular degeneration. Retina. 2011; 31(3):518-26. [PubMed: 21150696]

52. Querques G, Canoui-Poitrine F, Coscas F, et al. Analysis of progression of reticular pseudodrusen by spectral domain-optical coherence tomography. Invest Ophthalmol Vis Sci. 2012; 53(3):126470. [PubMed: 22266524]

53. De Bats F, Mathis T, Mauget-Faysse M, et al. Prevalence of Reticular Pseudodrusen in AgeRelated Macular Degeneration Using Multimodal Imaging. Retina. 2015

54. Wu Z, Ayton LN, Makeyeva G, et al. Impact of reticular pseudodrusen on microperimetry and multifocal electroretinography in intermediate age-related macular degeneration. Invest Ophthalmol Vis Sci. 2015; 56(3):2100-6. [PubMed: 25736790]

55. Klein R, Klein BE, Linton KL. Prevalence of age-related maculopathy. The Beaver Dam Eye Study Ophthalmology. 1992; 99(6):933-43. [PubMed: 1630784]

56. Boddu S, Lee MD, Marsiglia M, et al. Risk factors associated with reticular pseudodrusen versus large soft drusen. Am J Ophthalmol. 2014; 157(5):985-93 e2. [PubMed: 24491417]

57. Hogg RE, Silva R, Staurenghi G, et al. Clinical characteristics of reticular pseudodrusen in the fellow eye of patients with unilateral neovascular age-related macular degeneration. Ophthalmology. 2014; 121(9):1748-55. [PubMed: 24856310]

58. Lee MY, Yoon J, Ham DI. Clinical characteristics of reticular pseudodrusen in Korean patients. Am J Ophthalmol. 2012; 153(3):530-5. [PubMed: 21996310]

59. Spaide RF. Colocalization of pseudodrusen and subretinal drusenoid deposits using high-density en face spectral domain optical coherence tomography. Retina. 2014; 34(12):2336-45. [PubMed: 25380066]

60. Vongkulsiri S, Ooto S, Mrejen S, et al. The lack of concordance between subretinal drusenoid deposits and large choroidal blood vessels. Am J Ophthalmol. 2014; 158(4):710-5. [PubMed: 25034112]

61. Vongkulsiri S, Suzuki M, Spaide RF. Colocalization error between the scanning laser ophthalmoscope infrared reflectance and optical coherence tomography images of the heidelberg spectralis. Retina. 2015; 35(6):1211-5. [PubMed: 25748282]

62. Querques G, Querques L, Forte R, et al. Choroidal changes associated with reticular pseudodrusen. Invest Ophthalmol Vis Sci. 2012; 53(3):1258-63. [PubMed: 22222508] 
63. Grewal DS, Chou J, Rollins SD, Fawzi AA. A pilot quantitative study of topographic correlation between reticular pseudodrusen and the choroidal vasculature using en face optical coherence tomography. PLoS One. 2014; 9(3):e92841. [PubMed: 24658298]

64. Yoneyama S, Sakurada Y, Mabuchi F, et al. Genetic and clinical factors associated with reticular pseudodrusen in exudative age-related macular degeneration. Graefes Arch Clin Exp Ophthalmol. 2014; 252(9):1435-41. [PubMed: 24595987]

65. Eliasieh K, Liets LC, Chalupa LM. Cellular reorganization in the human retina during normal aging. Invest Ophthalmol Vis Sci. 2007; 48(6):2824-30. [PubMed: 17525218]

66. Curcio CA. Photoreceptor topography in ageing and age-related maculopathy. Eye (Lond). 2001; 15(Pt 3):376-83. [PubMed: 11450761]

67. Curcio CA, Millican CL, Allen KA, Kalina RE. Aging of the human photoreceptor mosaic: evidence for selective vulnerability of rods in central retina. Invest Ophthalmol Vis Sci. 1993; 34(12):3278-96. [PubMed: 8225863]

68. Ach T, Tolstik E, Messinger JD, et al. Lipofuscin redistribution and loss accompanied by cytoskeletal stress in retinal pigment epithelium of eyes with age-related macular degeneration. Invest Ophthalmol Vis Sci. 2015; 56(5):3242-52. [PubMed: 25758814]

69. Ach T, Huisingh C, McGwin G Jr, et al. Quantitative autofluorescence and cell density maps of the human retinal pigment epithelium. Invest Ophthalmol Vis Sci. 2014; 55(8):4832-41. [PubMed: 25034602]

70. Curcio CA, Messinger JD, Sloan KR, et al. Human chorioretinal layer thicknesses measured in macula-wide, high-resolution histologic sections. Invest Ophthalmol Vis Sci. 2011; 52(7):394354. [PubMed: 21421869]

71. Ramrattan RS, van der Schaft TL, Mooy CM, et al. Morphometric analysis of Bruch's membrane, the choriocapillaris, and the choroid in aging. Invest Ophthalmol Vis Sci. 1994; 35(6):2857-64. [PubMed: 8188481]

72. Sohn EH, Flamme-Wiese MJ, Whitmore SS, et al. Loss of CD34 expression in aging human choriocapillaris endothelial cells. PLoS One. 2014; 9(1):e86538. [PubMed: 24466138]

73. Khandhadia S, Cipriani V, Yates JR, Lotery AJ. Age-related macular degeneration and the complement system. Immunobiology. 2012; 217(2):127-46. [PubMed: 21868123]

74. Skeie JM, Fingert JH, Russell SR, et al. Complement component C5a activates ICAM-1 expression on human choroidal endothelial cells. Invest Ophthalmol Vis Sci. 2010; 51(10):5336-42. [PubMed: 20484595]

75. Bhutto IA, Baba T, Merges C, et al. C-reactive protein and complement factor $\mathrm{H}$ in aged human eyes and eyes with age-related macular degeneration. Br J Ophthalmol. 2011; 95(9):1323-30. [PubMed: 21633121]

76. Dashti N, McGwin G, Owsley C, Curcio CA. Plasma apolipoproteins and risk for age related maculopathy. Br J Ophthalmol. 2006; 90(8):1028-33. [PubMed: 16723359]

77. Pumariega NM, Smith RT, Sohrab MA, et al. A prospective study of reticular macular disease. Ophthalmology. 2011; 118(8):1619-25. [PubMed: 21550118]

78. Tserentsoodol N, Gordiyenko NV, Pascual I, et al. Intraretinal lipid transport is dependent on high density lipoprotein-like particles and class B scavenger receptors. Mol Vis. 2006; 12:1319-33. [PubMed: 17110915]

79. Curcio CA, Sloan KR, Kalina RE, Hendrickson AE. Human photoreceptor topography. J Comp Neurol. 1990; 292(4):497-523. [PubMed: 2324310]

80. Hoffmann EM, Zangwill LM, Crowston JG, Weinreb RN. Optic disk size and glaucoma. Surv Ophthalmol. 2007; 52(1):32-49. [PubMed: 17212989]

81. Curcio CA, Saunders PL, Younger PW, Malek G. Peripapillary chorioretinal atrophy: Bruch's membrane changes and photoreceptor loss. Ophthalmology. 2000; 107(2):334-43. [PubMed: 10690836]

82. Schaal KB, Legarreta AD, Gregori G, et al. Widefield En Face Optical Coherence Tomography Imaging of Subretinal Drusenoid Deposits. Ophthalmic Surg Lasers Imaging Retina. 2015; 46(5): 550-9. [PubMed: 26057758] 


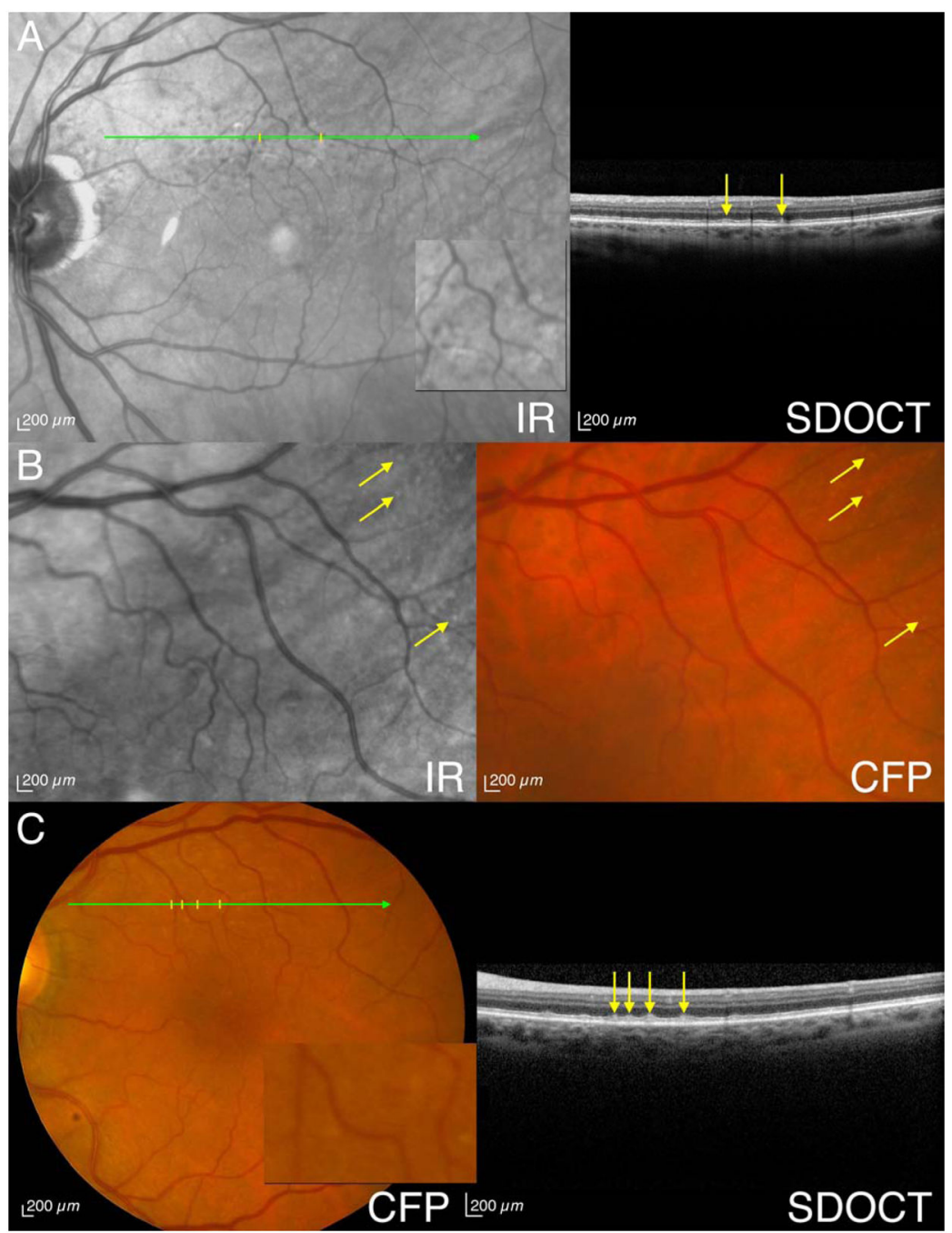

Figure 1. Eyes meeting strict criteria for subretinal drusenoid deposits (SDD) with multimodal imaging

A. An 81-year-old man (Age-Related Eye Disease Study [AREDS] grade 4) has visible SDD by infrared reflectance (IR) (indicated by ticks; magnified $3 \mathrm{X}$ in inset), spectral domain optical coherence tomography (SD-OCT) (arrows) and fundus autofluorescence (FAF) (not shown). B. A 73-year-old woman (AREDS grade 1) has visible SDD by IR (arrows) and color fundus photographs (CFP) (arrows) without SD-OCT findings (not shown). C. A 78year-old woman (AREDS grade 2) has visible SDD by CFP (indicated by ticks; magnified $3 \mathrm{X}$ in inset), SD-OCT (arrows) and IR (not shown). 




Figure 2. Non-age-related macular degeneration (AMD) subject with subretinal drusenoid deposits (SDD) by strict criteria

A-C. A 66-year-old woman (Age-Related Eye Disease Study [AREDS] grade 1).. A. An infrared reflectance (IR) image of a healthy macula. B. When the IR image is magnified, a hyporeflective smudge (arrowhead) that correlates with optical coherence tomography (OCT) scan in C is apparent. C. By spectral domain (SD) OCT, hyperreflective material between retinal pigment epithelium (RPE) and ellipsoid zone (arrowhead) is classified as SDD (stage 2). This lesion was undetectable on fundus autofluorescence (FAF) and color fundus photographs (CFP) (not shown). 


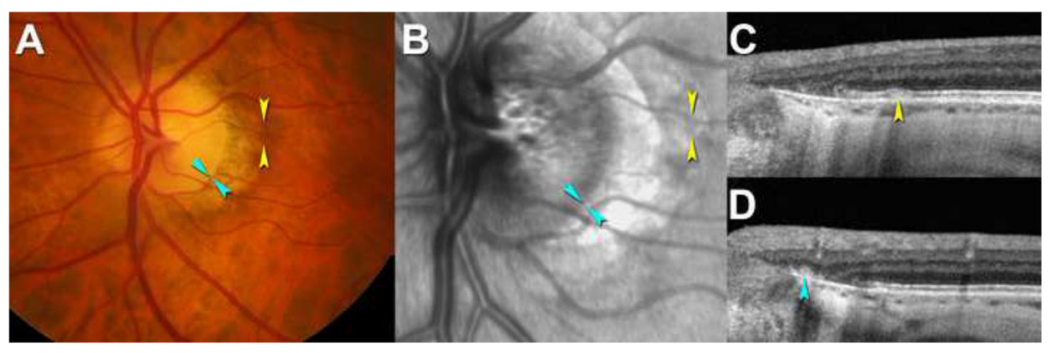

Figure 3. Peripapillary subretinal drusenoid deposits (SDD) by strict criteria A-D. A 70-year-old man (Age-Related Eye Disease Study [AREDS] grade 1). A. color fundus photograph (CFP) shows a healthy macula. Arrowheads indicate locations where SDD (yellow) and basal laminar deposit (cyan) was found on optical coherence tomography (OCT) scans in C. B. Mottled reflectivity seen on infrared reflectance (IR) (yellow) was not seen on fundus autofluorescence (FAF) (not shown). Site of peripapillary basal laminar deposit is shown (cyan). C. SDD is seen in the optic nerve head (ONH) spectral domain (SD) OCT volume as a hyperreflective mound between retinal pigment epithelium (RPE) and ellipsoid zone (yellow). D. Basal laminar deposit without overlying RPE (cyan) is close to the termination of Bruch's membrane and reflective. 
Table 3

Demographic, lifestyle and medical characteristics stratified by subretinal drusenoid deposit presence at the person level using strict criteria

\begin{tabular}{|c|c|c|c|}
\hline & SDD, N (\%) & No SDD, $N(\%)$ & p-value \\
\hline Total sample & $197(32.2)$ & $414(67.8)$ & -- \\
\hline \multicolumn{4}{|l|}{ Age group, years } \\
\hline $60-69$ & $98(27.1)$ & $264(72.9)$ & \multirow[t]{3}{*}{$<0.000$} \\
\hline $70-79$ & $78(36.5)$ & $136(63.5)$ & \\
\hline 280 & $21(60.0)$ & $14(40.0)$ & \\
\hline \multicolumn{4}{|l|}{ Gender } \\
\hline Female & $121(30.6)$ & $274(69.4)$ & \multirow[t]{2}{*}{0.25} \\
\hline Male & $76(35.2)$ & $140(64.8)$ & \\
\hline \multicolumn{4}{|l|}{ Race } \\
\hline Non-White & $6(20.0)$ & $24(80.0)$ & \multirow[t]{2}{*}{0.14} \\
\hline White & $191(32.9)$ & $390(67.1)$ & \\
\hline \multicolumn{4}{|l|}{ Education } \\
\hline High school or less & $39(30.2)$ & $90(69.8)$ & \multirow[t]{2}{*}{0.58} \\
\hline Some college or more & $158(32.8)$ & $324(67.2)$ & \\
\hline \multicolumn{4}{|l|}{ Smoking status } \\
\hline Current & $8(26.7)$ & $22(73.3)$ & \multirow[t]{3}{*}{0.41} \\
\hline Former & $76(30.0)$ & $177(70.0)$ & \\
\hline Never & $113(34.6)$ & $214(65.4)$ & \\
\hline \multicolumn{4}{|c|}{ Alcohol use, drinks per week } \\
\hline Abstainers & $60(29.7)$ & $142(70.3)$ & \multirow[t]{4}{*}{0.80} \\
\hline Light & $56(32.8)$ & $115(67.2)$ & \\
\hline Moderate & $63(34.4)$ & $120(65.6)$ & \\
\hline Heavy & $18(32.7)$ & $37(67.3)$ & \\
\hline \multicolumn{4}{|c|}{ Cardiovascular disease, self-reported } \\
\hline Yes & $68(35.2)$ & $125(64.8)$ & \multirow[t]{2}{*}{0.28} \\
\hline No & $128(30.8)$ & $287(69.2)$ & \\
\hline \multicolumn{4}{|l|}{ High blood pressure } \\
\hline Yes & $110(34.7)$ & $207(65.3)$ & \multirow[t]{2}{*}{0.18} \\
\hline No & $86(29.6)$ & $205(70.4)$ & \\
\hline \multicolumn{4}{|l|}{ Statin use } \\
\hline Yes & $34(27.9)$ & $88(72.1)$ & \multirow[t]{2}{*}{0.25} \\
\hline No & $163(33.3)$ & $326(66.7)$ & \\
\hline
\end{tabular}

Abbreviation: SDD = subretinal drusenoid deposits 


\section{Table 4}

Subretinal drusenoid deposit presence by strict criteria at the person level, stratified by age-related macular degeneration status (AREDS 1-8)

\begin{tabular}{|l|r|r|r|r|}
\hline \multirow{2}{*}{} & \multicolumn{2}{|c|}{ SDD } & \multicolumn{2}{c|}{ No SDD } \\
\cline { 2 - 5 } & $\mathbf{N}$ & $\%$ & $\mathbf{N}$ & $\mathbf{\%}$ \\
\hline Total sample $^{a}$ & 196 & 32.3 & 411 & 67.7 \\
\hline AREDS, grade $^{*}$ & & & & \\
\hline 1 & 95 & 23.0 & 318 & 77.0 \\
\hline 2 & 47 & 46.1 & 55 & 53.9 \\
\hline 3 & 16 & 59.3 & 11 & 40.7 \\
\hline 4 & 23 & 50.0 & 23 & 50.0 \\
\hline 5 & 5 & 62.5 & 3 & 37.5 \\
\hline 6 & 5 & 83.3 & 1 & 16.7 \\
\hline 7 & 4 & 100.0 & 0 & 0 \\
\hline 8 & 1 & 100.0 & 0 & 0 \\
\hline
\end{tabular}

Abbreviations:

${ }^{a}$ Data for 4 participants are unavailable because of incomplete AREDS grading; SDD = subretinal drusenoid deposits; AREDS = Age-Related Eye Disease Study. 


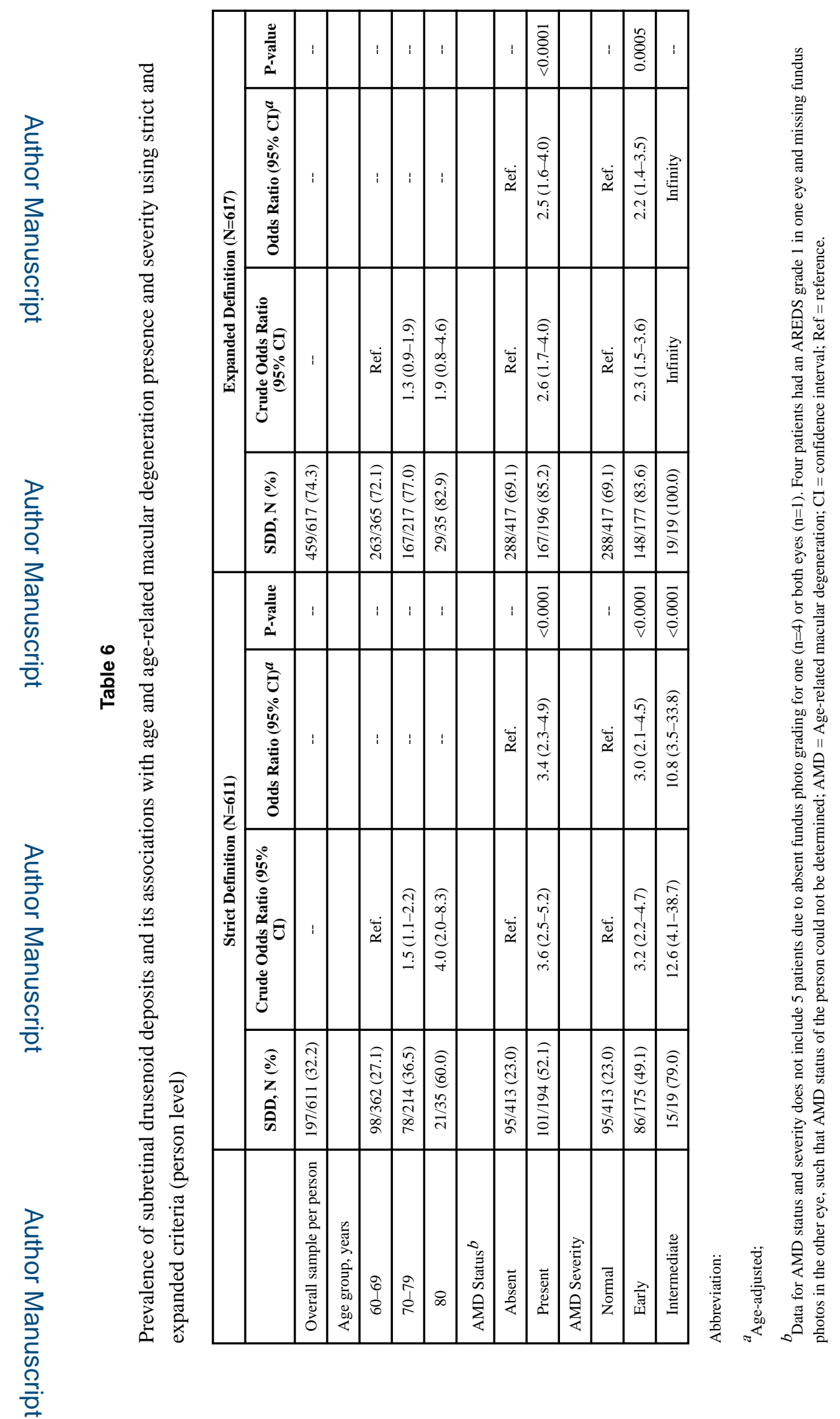

Ophthalmology. Author manuscript; available in PMC 2017 May 01. 